\title{
Megalencephalic Leukoencephalopathy with Subcortical Cyst: A Case Report
}

\author{
Gangadhar K, Patwari S, Verma A, Kaviyarasy
}

Department of Radiodiagnosis and imaging, Institute of Medical Sciences, Banaras Hindu University, Varanasi, India

\begin{abstract}
Megalencephalic leukoencephalopathy with subcortical cysts (MLC) is a rare disease first described by van der Knaap et al, in 1995. MLC is a relatively new entity of neurodegenerative disorder of autosomal recessive inheritance characterized by infantile onset macrocephaly, cerebral leucoencephalopathy, mild neurological symptoms and an extremely slow course of functional deterioration. The degree of macrocephaly is variable and can be as much as 4-6 SD above the mean. Almost all patients have seizures from an early age. Some patients have died in their 2 nd and 3rd decades but few may live till fourth decade. We report a case of 5-year-old girl diagnosed to have this disease.
\end{abstract}

Keywords: Macrocephaly, Megalencephalic leukoencephalopathy, Subcortical cysts

\section{Case Report}

A 5-year-old girl, belonging to BansalAggarwal community in northern India born of non-consanguineous marriage presented with large head, seizures, mental retardation, delayed mental and motor milestones, and unsteadiness of gait. There was no history of seizures or myoclonic jerks. Family history was negative. Examination revealed short stature, macrocrania (head circumference $70 \mathrm{~cm}$ exceeding the 95 th percentile for age), mild cognitive impairment, no features of cranial nerve deficits with normal bilateral deep tendon reflexes and plantar response. Ocular, chest and abdominal examinations were unremarkable.

Magnetic resonance imaging of brain

Correspondence to: Dr. Kiran Gangadhar Department of Radiodiagnosis and imaging, Institute Of Medical Sciences, Banaras Hindu University, Varanasi . India Email: kirang.585@googlemail.com
(T1 weighted, Fluid Attenuated Inversion Recovery (FLAIR), T2 Weighted, diffusion weighted, post contrast T1 weighted and Phase sensitive inversion recovery images) showed diffuse altered supratentorial white matter signal intensities appearing hypointense on T1W, and hyperintense on FLAIR and T2W. In addition, large welldefined subcortical cysts were noted in bilateral anterior temporal lobes (Figs. 1-6). There was sparing of internal capsule, corpus callosum and brain stem. MR spectroscopic traces showed relatively preserved and slightly lower NAA and mildly elevated choline on intermediate TE.

\section{Discussion}

Megalencephalic leukoencephalopathy with subcortical cysts was first described by van der Knaap et al. in 1995. MLC is a rare disease with a low carrier rate. The disease has a high incidence in Agarwal community in India and Jewish community with consanguinity being a high risk factor. ${ }^{2-6}$ 

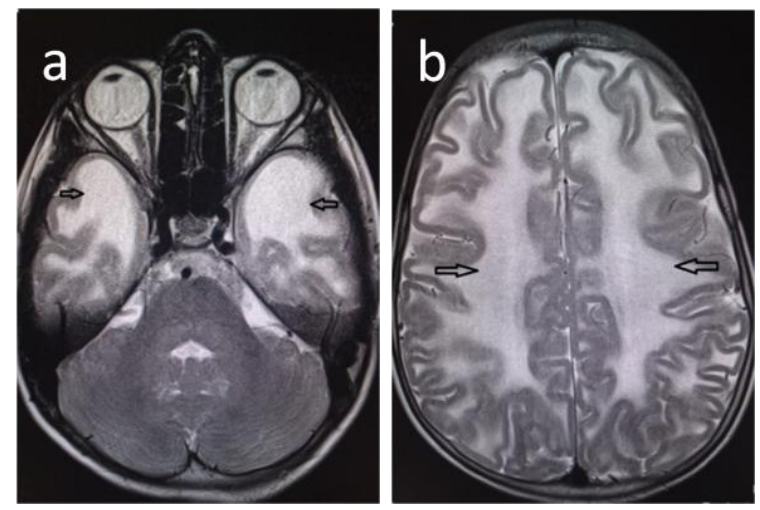

Fig 1: Five year old girl with diagnosis of Van Der Knaap disease. Axial T2 weighted (TR-4341/TE-90) magnetic resonance imaging of brain with 1.5 Tesla Magnetom Avanto, showing macrocephaly, intraparenchymal subcortical cysts (open arrows) in B/L temporal lobes with adjacent temporal lobe white matter hyperintensity (fig a) and bilateral symmetrical white matter hyperintensity (open arrows) suggestive of demyelination noted involving corona radiata and subcortical ' $U$ ' fibres ( fig b).

MLC is an autosomal recessive disorder due to mutations in MLC 1 gene $^{7}$ which has its locus in chromosome 22qtel. The physiological function of the protein is at present unknown. It is probably an integral membrane protein. The diagnosis of MLC can be made with confidence in patients with typical clinical findings and characteristic abnormalities on cranial MRI. MLC is known for its mild neurological signs and symptoms in the setting of very abnormal MR findings. Macrocephaly is present at birth or, more commonly, develops within the first year of life in all patients. Almost all patients have seizures from an early age. Early development is normal or mildly delayed. Slow deterioration of motor functions with cerebellar ataxia and mild spasticity usually starts in early childhood. Some patients have extrapyramidal movement abnormalities with dystonia and athetosis, usually as a late finding. Mental decline occurs later and is much milder than motor decline. ${ }^{8}$ In typical cases, the MRI findings are often diagnostic of MLC, MRI shows 'swollen white matter'
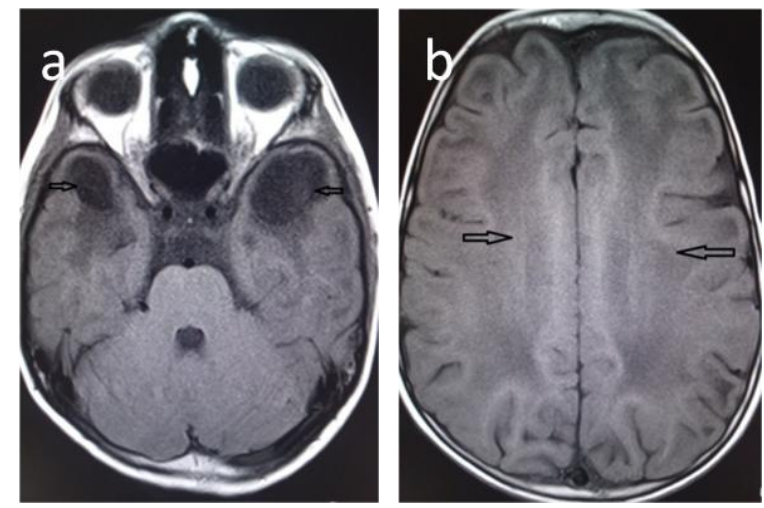

Fig 2: Five year old girl with diagnosis of Van Der Knaap disease. Axial T1 weighted (TR-550/TE-8.4) magnetic resonance imaging of brain with 1.5 Tesla Magnetom Avanto showing macrocephaly, intraparenchymal subcortical cysts (open arrows) in $B / L$ anterior temporal lobes (fig a) and bilateral symmetrical white matter hypointensity (open arrows) suggestive of demyelination (fig b).

and diffuse supratentorial symmetrical white matter changes in the cerebral hemispheres with relative sparing of central white matter structures like the corpus callosum, internal capsule, and brain stem. Subcortical cysts are almost always present in the anterior temporal region and are also frequently noted in the frontoparietal region. Grey matter is usually spared. Gradually the white matter swelling decreases and cerebral atrophy may ensue. The subcortical cysts may increase in size and number.

The differential diagnosis of MLC includes, Alexander disease, Cystic leukoencephalopathy without megalencephaly, Vanishing white matter disease, Canavan's disease and Glutaric aciduria type 1 . These conditions have relentlessly progressive infantile onset leukoencephalopathy that is frequently fatal within first decade of life, however MLC has remarkably slow course of deterioration in neurologic function. MLC must be included in differential diagnosis of macrocephaly 

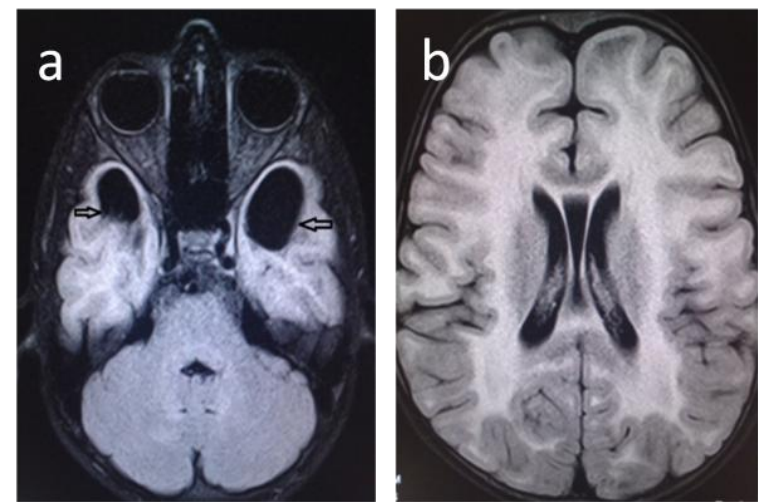

Fig 3: Five year old girl with diagnosis of Van Der Knaap disease. Axial FLAIR (TR-9000/TE-89) magnetic resonance imaging of brain with 1.5 Tesla Magnetom Avanto, showing intra parenchymal subcortical cysts (open arrows) in $\mathrm{B} / \mathrm{L}$ anterior temporal lobes revealing fluid suppression with adjacent temporal lobe white matter hyperintensity (fig a) with sparing of brain stem and bilateral symmetrical white matter hyperintensity suggestive of demyelination involving periventricular white matter, centrum semiovale and subcortical ' $U$ ' fibres ( fig b).
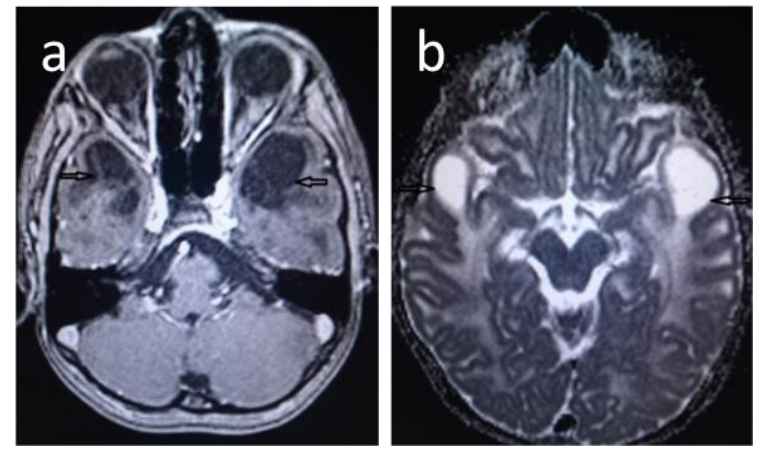

Fig 5a: Five year old girl with diagnosis of Van Der Knaap disease. Axial T1 weighted post gadolinium (TR-550/TE-8.4) magnetic resonance imaging of brain with 1.5 Tesla Magnetom Avanto, showing nonenhancing intraparenchymal subcortical cysts (open arrows) in $\mathrm{B} / \mathrm{L}$ anterior temporal lobes. Fig 5b) ADC maps showing facilitated diffusion in both sub cortical cysts (open arrows) in temporal lobes and adjacent temporal white matter.
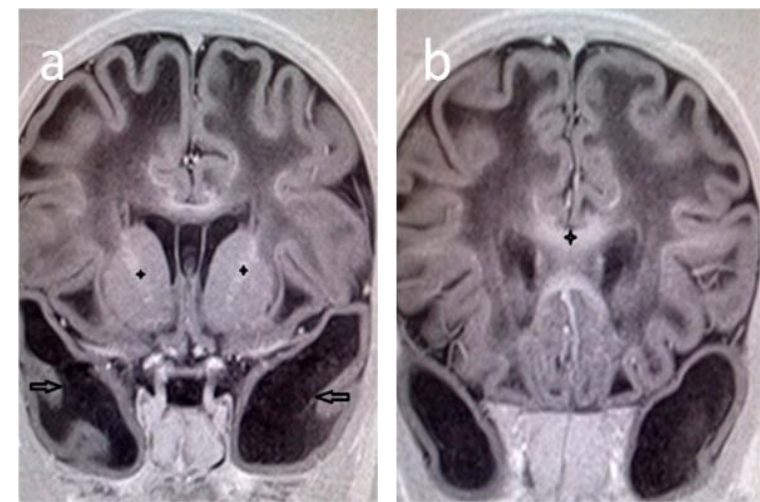

Fig 4: Five year old girl with diagnosis of Van Der Knaap disease. Coronal Phase sensitive inversion recovery (TR-7000/TE69) magnetic resonance imaging of brain with 1.5 Tesla Magnetom Avanto, showing diffuse symmetrical white matter hypointensity with sparing of internal capsule(stars) and temporal lobe subcortical cysts (open arrows)( fig a). Fig 4b) clearly showing sparing of corpus callosum.

with early onset leukoencephalopathy. ${ }^{7}$ In Alexander disease frontal lobe white matter involvement with post contrast enhancement is seen and also parietal white matter, internal and external capsules and basal ganglia may be involved. In vanishing white matter disease extensive symmetric CSF-isointense white matter changes and radiating cystic degeneration would be seen. In Cystic leukoencephalopathy without megalencephaly bilateral temporal subcortical cysts and multifocal lobar white matter lesions showing hypointensity on T1W and hyperintensity on T2W and FLAIR with central white matter sparing will be seen. In Canavans disease diffuse involvement (including subcortical U fibers) of white matter with markedly increased NAA on MR spectroscopy noted. In glutaric aciduria type I frontotemporal atrophy, "bat wing" dilatation of Sylvian fissures, increased $\mathrm{T} 2 \mathrm{~W}$ intensity in bilateral caudate 
nuclei, putamen and delay in myelination is seen.

Table 1: Summary table of Megalencephalic leukoencephalopathy with subcortical cysts.

\begin{tabular}{|c|c|}
\hline Etiology & $\begin{array}{l}\text { A autosomal recessive } \\
\text { disorder due to mutations in } \\
\text { MLC1 gene on chr22qtel }\end{array}$ \\
\hline Incidence & $\begin{array}{l}\text { MLC is a rare disease with a } \\
\text { low carrier rate. The disease has } \\
\text { a high incidence in populations } \\
\text { in which consanguinity is } \\
\text { common }\end{array}$ \\
\hline $\begin{array}{l}\text { Gender } \\
\text { ratio }\end{array}$ & No gender predilection proved \\
\hline $\begin{array}{l}\text { Age } \\
\text { predilection }\end{array}$ & $\begin{array}{l}\text { The age at onset of symptoms } \\
\text { varied from birth to } 25 \text { years }\end{array}$ \\
\hline Risk factors & Consanguinity \\
\hline Treatment & $\begin{array}{l}\text { All attempts to treat MLC have } \\
\text { failed. } \\
\text { Patients have been treated with } \\
\text { acetazolamide, but neither the } \\
\text { clinical symptoms nor the white } \\
\text { matter swelling improved. } \\
\text { Supportive therapy includes the } \\
\text { prescription of anticonvulsants } \\
\text { if the patient has seizures. } \\
\text { Physical therapy is important to } \\
\text { improve motor dysfunction. } \\
\text { Special education is required } \\
\text { for many patients. }\end{array}$ \\
\hline Prognosis & $\begin{array}{l}\text { Relentlessly progressive course } \\
\text { and is frequently fatal }\end{array}$ \\
\hline $\begin{array}{l}\text { Findings on } \\
\text { imaging }\end{array}$ & $\begin{array}{l}\text { Bilateral extensive } \\
\text { supratentorial white matter } \\
\text { involvement with temporal } \\
\text { subcortical cysts; relative } \\
\text { sparing of corpus callosum and } \\
\text { internal capsule }\end{array}$ \\
\hline
\end{tabular}

\section{Conclusion}

Megalencephalic leukoencephalopathy with subcortical cysts/ van der Knaap disease is the most common leukodystrophy with megalencephaly observed in India and should be considered in the differential diagnosis of children with megalencephaly and

leukoencephalopathy. Typical imaging findings are bilateral extensive supratentorial white matter involvement with temporal subcortical cysts and relative sparing of corpus callosum and internal capsule. One should suspect and carry-out genetic tests to confirm the diagnosis.

\section{References}

1. Van der Knaap MS, Barth PG, Stroink $\mathrm{H}$, et al. Leukoencephalopathy with swelling and a discrepantly mild clinical course in eight children. Ann Neurol1995; 37:324-34. PMID:7695231

2. Ben-Zeev B, Gross V, Kushnir T, et al. Vacuolating megalencephalic leukoencephalopathy in 12 Israeli patients. J Child Neurol 2001; 16: 939. PMID:11292232

3. Topcu M, Saatci I, Topcuoglu MA, et al. Megalencephaly and leukodystrophy with mild clinical course: a report on 12 new cases. Brain Dev 1998;20:142-53.PMID:9628190

4. Singhal BS, Gursahani RD, Udani VP et al. Megalencephalic leukodystrophy in an Asian Indian ethnic group. Pediatr Neurol 1996; 14:291-6. PMID:8805171

5. Gorospe JR et al Indian Agarwal megalencephalic leukodystrophy with cysts is caused by a common MLC1 mutation Neurology. 2004 Mar 23;62(6):878-82 PMID 15037685

6. Y Shinar et al ,A common ancestral haplotype in carrier chromosomes from different ethnic backgrounds in vacuolating megalencephalic leucoencephalopathy with subcortical cysts J Med Genet 2002;39:54-57 PMID: 11826027. 
7. Leegwater FA, Boor PK, Yuan BQ, et al. Identification of novel mutations in Megalencephalic Leukoencephalopathy with Subcortical Cysts responsible for megalencephalic leukoencephalopathy with subcortical cysts. Hum Genet 2002; 110: 279-83.
8. KV Rajagopal et al. Van der Knaap Disease, A Megalencephalic Leukoencephalopathy. Ind $\mathrm{J}$ Radiol Imag 2006; 16:4:733-734. 